\title{
COMPARACIÓN DEL ACV DE 2 ESTIBAS CONSTRUIDAS CON MADERA NATURAL Y MADERA PLÁSTICA EN SIMAPRO 8.4.0
}

\author{
Karen Dayana Fuentes Fuentes ${ }^{1}$, Angélica Patricia Vanegas Padilla ${ }^{2}$, Karina Paola Torres Cervera ${ }^{3}$.
}

1'Ingeniera Ambiental y Sanitario. Valledupar, Colombia. karen01-23@hotmail.com

${ }^{2}$ Ingeniera Ambiental y Sanitario, Magíster en Desarrollo Sostenible y Medio Ambiente, Esp. Gestión Ambiental. Docente Universidad Popular del Cesar. Valledupar, Colombia. angelicavanegasp@unicesar.edu.co https://orcid.org/0000-0001-5087-6172

${ }^{3}$ Ingeniera Ambiental y Sanitario, Magíster en Medio ambiente y Desarrollo. Docente Universidad Popular del

Cesar. Valledupar karinaptorres@unicesar.edu.co

\section{RESUMEN}

El objetivo del estudio refleja una comparación del análisis de ciclo de vida conceptual para 2.00 estibas construidas con madera natural (melinna) y madera plástica (100\% PET), usando el software SIMAPRO 8.4.0 en su versión DEMO 2017 bajo la norma la UNE-EN ISO 14040. La metodología utilizada fue experimental y descriptiva donde hubo manipulación de variables tales como: resistencia a la flexión, contenido de humedad y durabilidad natural a agentes degradantes para la determinación de aspectos sociales, económicos y ambientales. Los resultados arrojados del análisis comparativo afirmaron que las estibas de madera natural afectan en un $100 \%$ a la salud humana, a diferencia de las estibas de madera plástica, las cuales afectan un $2 \%$ la vida en los ecosistemas. Para la conservación de la Biodiversidad, las estibas naturales afectan en un $100 \%$ y las estibas plásticas un $47 \%$. La madera plástica en comparación con la madera natural cuenta con varios beneficios como lo son la mayor impermeabilidad, fácil mantenimiento, fácil limpieza, mayor seguridad (por astillamiento) y baja absorción de humedad.

Palabras clave: Madera natural, madera plástica, PET, dureza, compresión, flexión.

Recibido: 01 de marzo de 2021. Aceptado: 20 de julio de 2021

Received: March 01, 2021. Accepted: July 20, 2021

DOI: https://doi.org/10.33571/rpolitec.v17n34a1

\section{COMPARISON OF THE LIFE CYCLE ANALYSIS OF 2 PALLETS BUILT WITH NATURAL WOOD AND PLASTIC WOOD RESPECTIVELY}

\begin{abstract}
The objective of the study reflects a comparison of the conceptual life cycle analysis for 2.00 pallets built with natural wood (melinna) and plastic wood (100\% PET), using the SIMAPRO 8.4.0 software in its DEMO 2017 version under the UNE standard -EN ISO 14040. The methodology used was experimental and descriptive where there was manipulation of variables such as: flexural strength, moisture content and natural durability to degrading agents for the determination of social, economic and environmental aspects. The results of the comparative analysis affirmed that the pallets of natural wood affect human health by $100 \%$, unlike the pallets of plastic wood, which affect life in ecosystems by $2 \%$. For the conservation of Biodiversity, natural pallets affect $100 \%$ and plastic pallets $47 \%$. Plastic wood, compared to natural wood, has several benefits such as greater impermeability, easy cleaning, greater safety (due to chipping), low moisture absorption.
\end{abstract}

Keywords: Natural wood, plastic wood, PET, hardness, compression, bending. 
Cómo citar este artículo: K.D. Fuentes, A.P. Vanegas, K.P. Torres. "Comparación del ACV de 2 estibas construidas con madera natural y madera plástica en Simapro 8.4.0", Revista Politécnica, vol.17, no.34 pp.9-29, 2021. DOI: https://doi.org/10.33571/rpolitec.v17n34a1

\section{INTRODUCCIÓN}

El desafío actual en las investigaciones de la ingeniería se focaliza en evitar o disminuir el daño latente que se produce al medio ambiente por los desechos asiduos de residuos que no son biodegradables tales como los plásticos (tardan en degradarse de 150 a 400 años), neumáticos, materiales sintéticos, entre otros. En el contexto de la construcción, se persigue modificar materiales como agregados, asfaltos, concretos, suelos y demás componentes utilizados en este sector con dichos materiales no biodegradables, con el objeto de seguir dando un uso específico, así ya hayan cumplido su periodo de vida útil.

La Organización de las Naciones Unidas (ONU) en el año 2018, dio a conocer que cada año se producen en el mundo más de 400 millones de toneladas de plástico y solo el $9 \%$ de los desperdicios son reciclados. Estos residuos, generados diariamente representan un contaminante notorio en el medio ambiente que traen consigo consecuencias negativas como lo son creación de plagas, calentamiento global, efecto invernadero, enfermedades, entre otros [2].

De acuerdo a lo anterior; surge la necesidad de proponer nuevas alternativas más ecológicas y sustentables para eliminar los impactos potenciales inferidos de la construcción de estibas naturales, dicha alternativa es la utilización de madera plástica para dicho fin, el cual se compone de polietileno $100 \%$ reciclado, el cual dará estibas con mejores propiedades físico-mecánicas que las naturales que a su vez reducirá considerablemente los residuos sólidos plásticos existentes en el área de implementación.

En la actualidad, es común el uso de estibas de madera natural como "cama" para el transporte de mercancía terrestre, esta actividad implica la extracción de cientos de árboles por día, ya que para construir 1 estiba se requieren aproximadamente 2 troncos de árboles y esto sería solo hablando de la extracción de materia prima dentro de todo el ciclo de vida de este [3].

Tras la implementación de este cambio de tal magnitud surge la necesidad de analizar el ciclo de vida de cada una de las estibas en estudio para así aportar conocimientos de primera mano sobre esta nueva alternativa ecológica, que hasta el momento ha sido bien aceptada por la industria del transporte de carga, conocer a profundidad y con detalles las actividades relacionadas con la construcción y vida útil de cada estiba, haciendo énfasis en su estadía sobre la tierra de "cuna a tumba". Saber cuáles componentes básicos (salud humana, ecosistemas y recursos) está siendo mayormente afectado para finalmente conocer cuál de las 2 estibas es más rentable ambientalmente.

Según lo anteriormente dicho, el objetivo de este artículo es comparar física y mecánicamente la madera plástica con la madera natural, con el fin de mostrar la viabilidad de su uso inicialmente en el sector constructivo a partir de la utilización de un software denominado SIMAPRO 8.4.0, en donde se elaboró el flujo de entradas y salidas a partir del análisis de inventario realizado con las actividades permitidas en SIMAPRO.

Posteriormente, se analizaron componentes individual y simultáneamente entre ambas estibas, y se justificó el porcentaje obtenido brevemente. Durante el estudio, se destacó la NO existencia de información oficial y pública sobre temas detallados sobre comparaciones de ACV entre dichas estibas [4].

\section{MATERIALES Y MÉTODO}


El tipo de investigación realizada fue experimental y descriptiva, hubo manipulación de variables tales como: resistencia a la flexión, contenido de humedad y durabilidad natural a agentes degradantes con condiciones rigurosamente controladas para el análisis de las conductas observadas en las muestras, descripción de cualidades y características sociales económicas y ambientales inherentes al proceso del ACV de las estibas de madera natural y plástica.

El diseño de la investigación de planificó y ejecutó en 5 etapas, organizadas metodológicamente de la siguiente manera: 1. Identificación de los puntos de generación de R.S.I en MERCAUPAR 2. Ensayos de las propiedades físicas y mecánicas de la madera natural y la madera plástica 3. Evaluación del ciclo de vida de las dos (2) maderas 4. Valoración económica (análisis de costos de producción) de los dos (2) tipos de estiba y 5 . Estudio de participación social.

La recolección del material que se utilizó para el muestreo de los ensayos de laboratorio se adquirió en la empresa MERCAUPAR S.A. Estas muestras se obtuvieron de varios locales de la empresa, sometidas a una transformación de madera natural para poder efectuar las pruebas que entregaran valores entre los rangos normales de variación de un material a partir de sus propiedades físico-mecánicas y de esta manera determinar si el rango de variación se encuentra en los límites de sus valores normales.

Las pruebas de caracterización física realizadas corresponden a densidad y dureza; en cuanto a las pruebas realizadas para caracterizar mecánicamente la madera plástica, se ejecutaron ensayos de resistencia a la flexotracción en sentido paralelo y resistencia a compresión en sentido perpendicular a la fibra y flexión.

\section{RESULTADOS}

Esta investigación se desarrolló en cinco etapas metodológicas con el fin de cumplir con los objetivos propuestos. La primera etapa fue la caracterización de los residuos sólidos, en la segunda etapa se analizaron los parámetros físicos mecánico de la madera natural y plástica, en la tercera etapa se evaluó el ciclo de vida de las estibas, posteriormente se realizó la valoración económica del ciclo de vida de las maderas y se culminó con un estudio de viabilidad social de las maderas

\subsection{Etapa $\mathrm{N}^{\circ}$ 1: Identificación de los puntos de generación de R.S.I en MERCAUPAR}

Las estibas de madera natural utilizadas en la investigación son conocidas en el medio comúnmente como Melina o Gmelina arbórea (nombre científico del árbol), estas fueron obtenida a través de la empresa maderas JORDAN HDR ubicados en Valledupar, Colombia. Véase Fig. 1. La madera plástica usada (100\% polietileno) se adquirió a través de la empresa MADERPLASTIC localizada en la ciudad de Cali, Colombia. Véase Fig. 2.

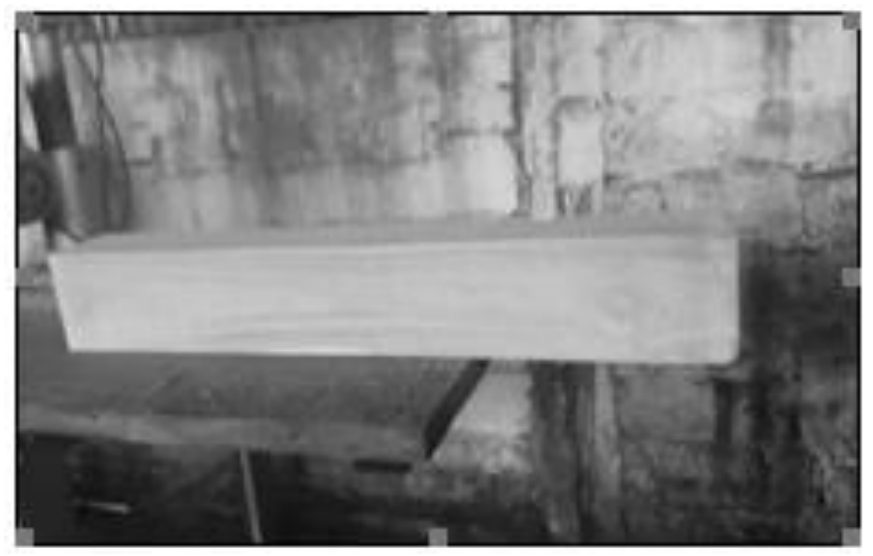

Fig. 1. Madera natural Melinna. 


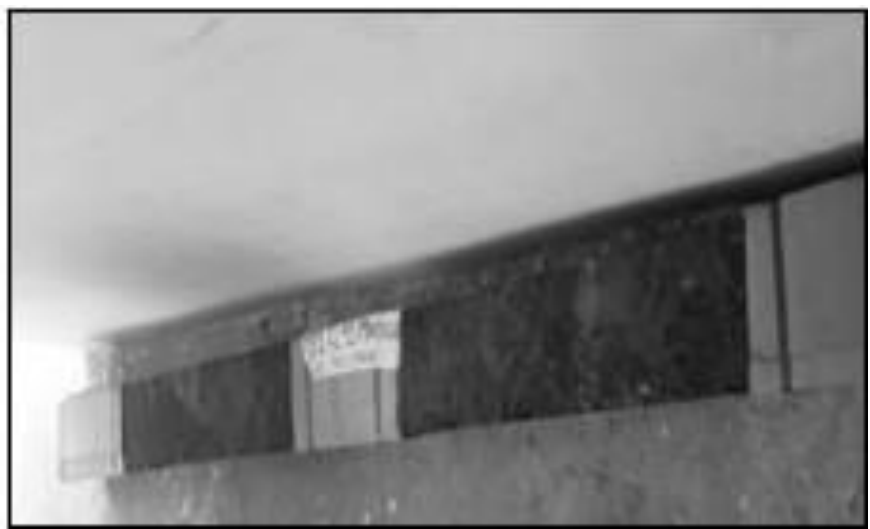

Fig. 2. Madera plástica, MADERPLASTIC.

\subsection{Etapa 2: Ensayos de las propiedades físicas y mecánicas de la madera natural y la madera} plástica

\subsubsection{Resistencia a la flexión bajo la NTC 663}

Teniendo en cuenta las especificaciones técnicas de la NTC 663 [5] aplicadas y usadas en el laboratorio de suelos y concreto a través de la máquina de flexión, cada muestra tuvo las siguientes dimensiones: Longitud: $45.5 \mathrm{~cm}$, Ancho: $15 \mathrm{~cm}$ y Profundidad: $4.00 \mathrm{~cm}$. Con el dato obtenido de esta prueba con valor promedio para las piezas de madera plástica de $2.2092 \%$ y $17.0373 \%$ para las piezas de madera natural se puede concluir que la madera para la prueba de flexión se encontraba en buen estado, ya que el porcentaje admisible de humedad para el ensayo según la NTC 663 está entre 12\% y 18\%. Véase las Fig. 3 y 4 que reflejan el ensayo de la resistencia a la flexotracción de las estibas de madera plástica y natural respectivamente.

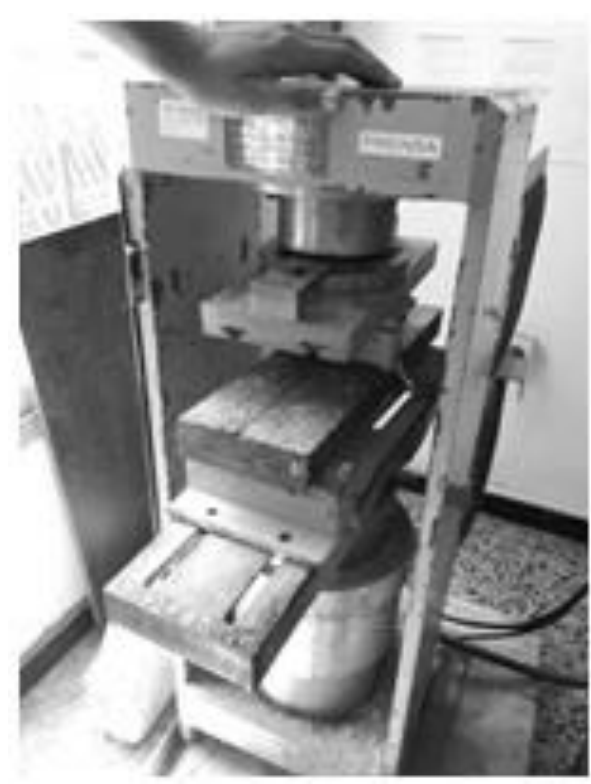

Fig. 3. Prueba de flexión estiba de madera plástica. 


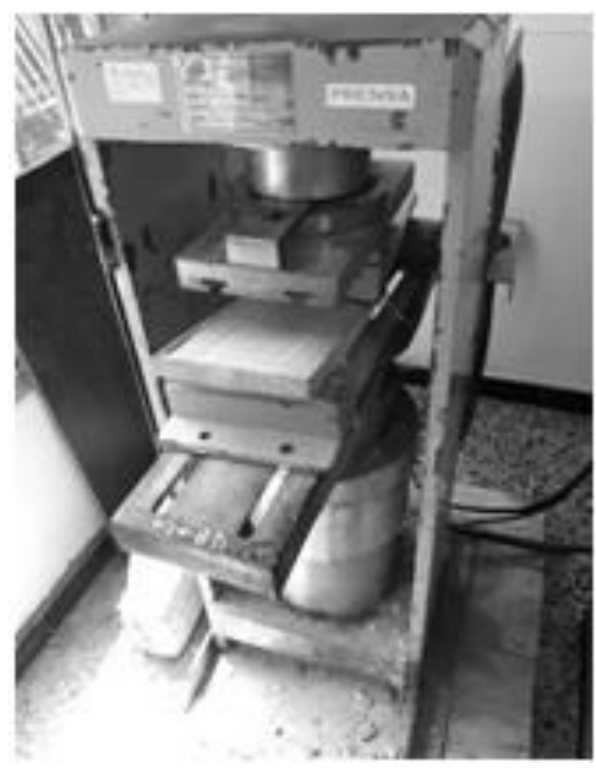

Fig. 4. Prueba de flexión estiba de madera natural.

Los resultados del laboratorio para el ensayo de flexión en la madera natural fueron para demostrar la veracidad de los siguientes valores arrojados en laboratorio.

La resistencia a la flexión de la madera es proporcional a su densidad, para confirmar esta afirmación, se compararon las resistencias de 5 especies más de madera natural, los valores encontrados fueron:

Tabla 1. Densidad y flexión máxima para varios tipos de madera natural.

\begin{tabular}{ccc}
\hline NOMBRE & $\begin{array}{c}\text { DENSIDAD SECA } \\
(\mathrm{KG} / \mathrm{CM} 3)\end{array}$ & $\begin{array}{c}\text { FLEXIÓN } \\
\text { MÁXIMA N/MM2 } \\
(\mathrm{MPA})\end{array}$ \\
\hline POCHOTE & 390 & 37,36 \\
\hline $\begin{array}{c}\text { MADERA MELINA } \\
\text { ENSAYO }\end{array}$ & 480 & 48,1 \\
\hline $\begin{array}{c}\text { MADERA MELINA } \\
\text { TEÓRICO }\end{array}$ & 480 & 49,32 \\
\hline CIPRÉS & 600 & 50,67 \\
\hline ACACIA & 610 & 66,3 \\
\hline ALGARROBO & 730 & 67,4 \\
\hline
\end{tabular}




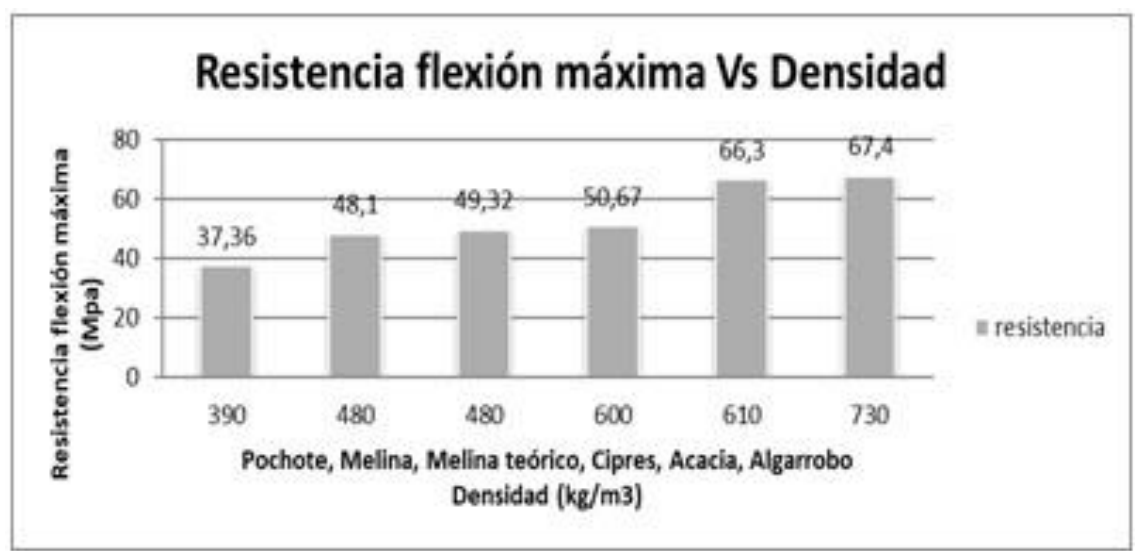

Fig. 5. Resistencia vs. Flexión en madera natural.

Esto nos confirma que la resistencia a la flexión de la madera melina, con respecto a su densidad, es muy buena. Se analizó lo mismo con la madera plástica, los resultados fueron:

Tabla 2. Densidad y flexión máxima para varios tipos de madera plástica.

\begin{tabular}{ccc}
\hline NOMBRE & $\begin{array}{c}\text { DENSIDAD SECA } \\
(\mathrm{KG} / \mathrm{CM} 3)\end{array}$ & $\begin{array}{c}\text { FLEXIÓN MÁXIMA } \\
\text { N/MM }\end{array}$ \\
\hline $\begin{array}{c}\text { MADERA PLÁSTICA } \\
\text { ENSAYO }\end{array}$ & 850 & 46,3 \\
\hline $\begin{array}{c}\text { MADERA PLÁSTICA } \\
\text { TEORIA 1 }\end{array}$ & 1101 & 28,5 \\
\hline $\begin{array}{c}\text { MADERA PLÁSTICA } \\
\text { TEORIA 2 }\end{array}$ & 1211 & 32,3 \\
\hline
\end{tabular}

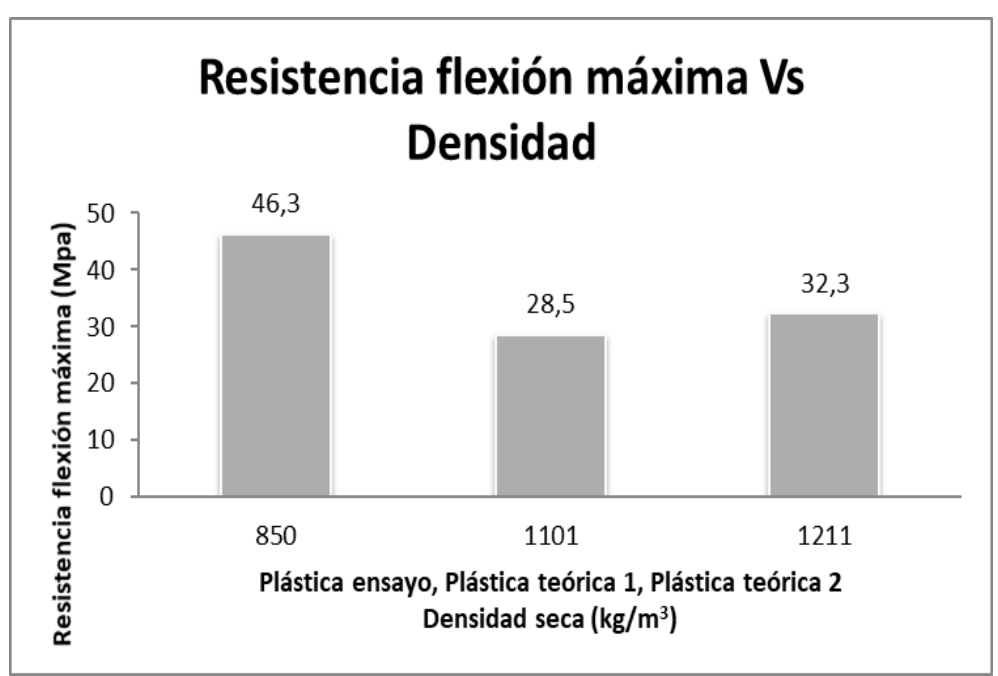

Fig. 6. Resistencia vs flexión en madera plástica.

Se evidenció que la madera plástica de estudio supera considerablemente los valores teóricos de otras piezas de madera plástica. Entonces, se califica como excelente, con respecto a su densidad. 
Finalmente, ambas maderas se clasifican como moderadamente resistentes a la flexión, sabiendo que la clasificación RESISTENTE es solo para aquella madera usada en construcción, las cuales superan los 500 $\mathrm{MPa}$.

\subsubsection{Ensayo contenido de humedad bajo la NTC 206-1}

Antes de iniciar cualquier tipo de prueba de laboratorio, dentro de la metodología se planteó verificar el contenido de humedad de cada una de las piezas de madera, para esto se tomó como modelo la norma técnica colombiana NTC 206-1 [6] (determinación del contenido de humedad para ensayos físicos y mecánicos).

Para esta prueba inicial se decidió tomar de cada una de las piezas de madera 5 probetas de sección $5 \times 4 \times 4 \mathrm{~cm}$, para total de 10 probetas. De las tres probetas se saca el promedio, de esta manera se obtiene el contenido de humedad por pieza utilizada en la investigación.

Se evaluaron condiciones de tiempo de retorno en unos periodos de retorno de 3.00 años. Al finalizar el procedimiento y tabulado los datos, se obtuvieron los siguientes valores para las piezas de madera.

Tabla 3. Resultados del ensayo de humedad en condiciones normales.

\begin{tabular}{cc}
\hline MADERA & MADERA \\
NATURAL & PLÁSTICA \\
\hline 19,3872 & 0,9166 \\
\hline 28,0559 & 0,9166 \\
\hline 29,5134 & 2,1625 \\
\hline 26,5484 & 2,2983 \\
\hline 19,791 & 1,6451 \\
\hline \multicolumn{2}{c}{ PROMEDIO } \\
\hline 24,65918 & 1,58782 \\
\hline
\end{tabular}

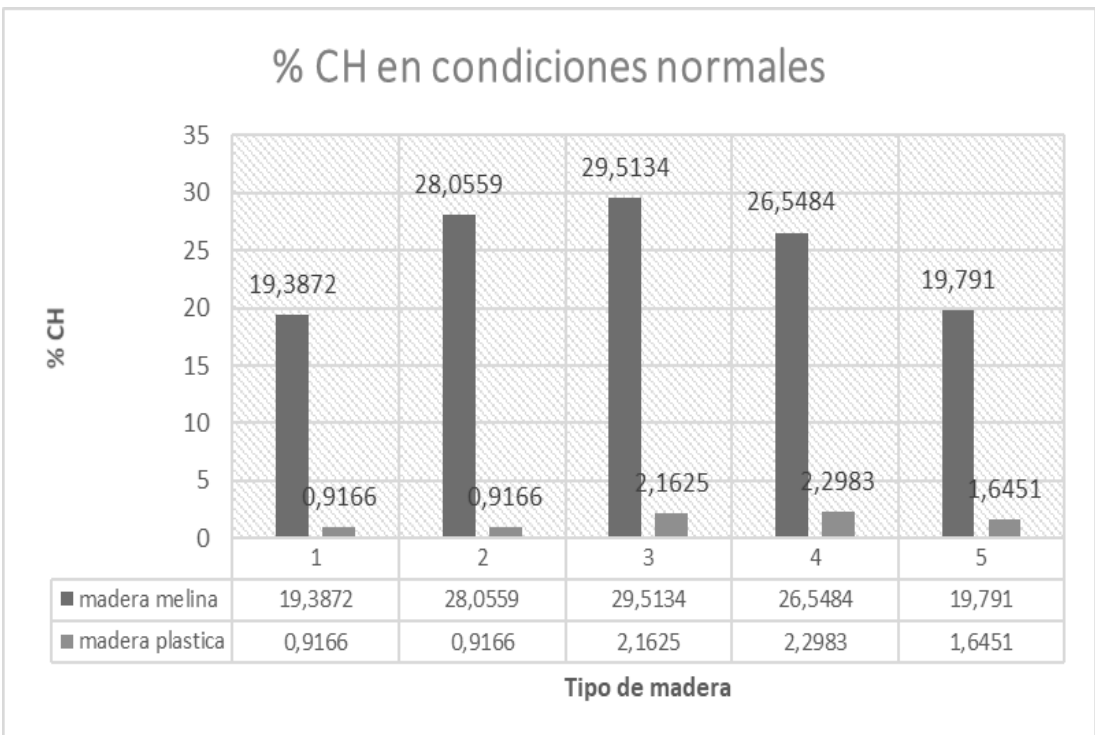

Fig. 7. \% Contenido de Humedad en condiciones normales. 
Se aprecia el notorio porcentaje de contenido de agua en las muestras de madera natural, llegando a un porcentaje promedio de $24,65918 \%$ y la madera plástica solo retiene un $1,58782 \%$, lo que demuestra que para condiciones normales las estibas de madera natural no cumplirían con el propósito de almacenamiento adecuado de alimentos y sus propiedades mecánicas ya se habrían reducido moderadamente debido a que su \%CH supera el estado muy seco.

Se determinó que la madera plástica para condiciones normales mantiene sus propiedades mecánicas, mantiene su estado en servicio, manteniendo la mercancía en excelentes condiciones. Al contrario que la madera natural.

Tabla 4. Resultados del ensayo de humedad en TR de 3 años.

\begin{tabular}{cc}
\hline $\begin{array}{c}\text { MADERA } \\
\text { NATURAL }\end{array}$ & $\begin{array}{c}\text { MADERA } \\
\text { PLAIICA }\end{array}$ \\
\hline 48,9958 & 0,8095 \\
\hline 50,1398 & 1,0617 \\
\hline 48,6501 & 1,7241 \\
\hline 48,8738 & 0,8564 \\
\hline 39,4444 & 1,4535 \\
\hline \multicolumn{2}{c}{ PROMEDIO } \\
\hline 47.2207 & 1.1810 \\
\hline \multicolumn{2}{c}{}
\end{tabular}

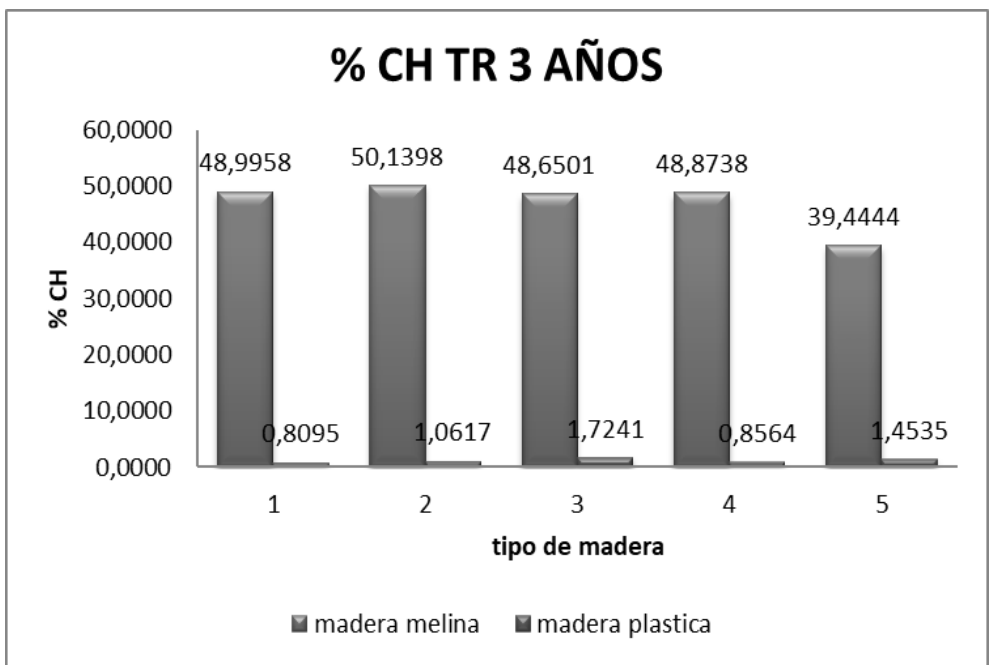

Fig. 8. Contenido de humedad en TR de 3 años.

Se evidenció el notorio porcentaje de contenido de agua en las muestras de madera natural, llegando a un porcentaje promedio de $47.2207 \%$ y la madera plástica solo retiene un $1.1810 \%$, lo que demuestra que para un tiempo de retorno de 3 años las estibas de madera natural no cumplirian con el propósito de almacenamiento adecuado de alimentos y sus propiedades mecánicas ya se habian reducido considerablemente debido al exceso de \%CH

Se concluye que la madera plástica para un tiempo de retorno de 3 años, permanece con sus propiedades mecánicas; mantiene su estado en servicio y la mercancía en excelentes condiciones, a diferencia de la madera natural [17]. 
Tabla 5. Resultados del ensayo de humedad para TR de 100 años.

\begin{tabular}{cc}
\hline MADERA & MADERA \\
NATURAL & PLÁSTICA \\
& \\
\hline 55,126 & 0,153 \\
\hline 57,9154 & 0,9296 \\
\hline 68,9062 & 0,9019 \\
\hline 78,3616 & 0,7224 \\
\hline 80,3528 & 1,53250 \\
\hline \multicolumn{2}{c}{ PROMEDIO } \\
\hline 68 & 0.84
\end{tabular}

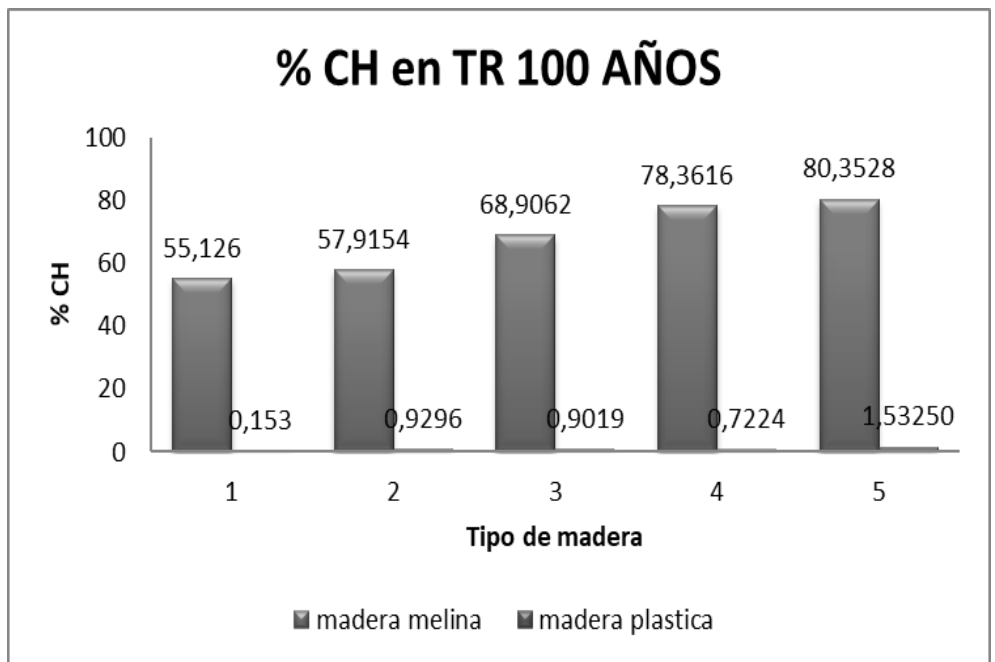

Fig. 9. Contenido de humedad en TR de 100 años.

Se aprecia que el \%CH de la madera natural para un tiempo de retorno de 100 años es mucho mas elevado que el $\% \mathrm{CH}$ de madera plastica, el \%CH maximo hallado es de $80,3528 \%$ lo que resulta lógico, sabiendo que la madera natural, en este caso la melina puede llegar a un $\% \mathrm{CH}$ de hasta el $120 \%$ de acuerdo a lo estipulado en la Norma Técnica Colombiana NTC 3545 [14].

La madera plástica sigue sin superar el $2 \%$ de contenido de humedad. Lo que la hace fielmente resistente a ataques de organismos xilófagos y mantiene sus propiedades mecánicas intactas.

\subsubsection{Ensayo según NTC 4132 (HONGOS XILÓFAGOS)}

Las láminas de madera natural fueron lijadas para extraer virutas de estas (véase Fig. 10), las de madera plástica fueron extraídas a mano de las láminas de madera, ya que se necesitaban piezas pequeñas para poder triturarlas [7]. (véase Fig. 10 y 11). 


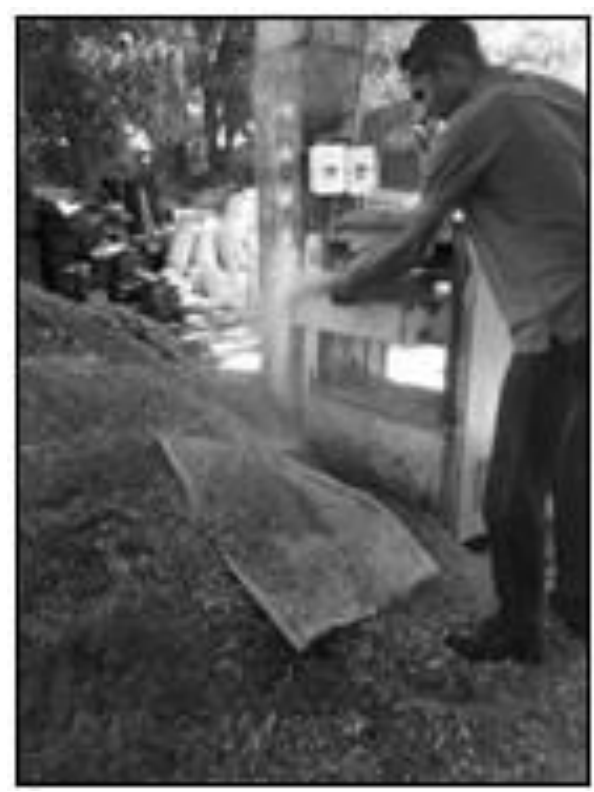

Fig. 10 Madera natural lijada

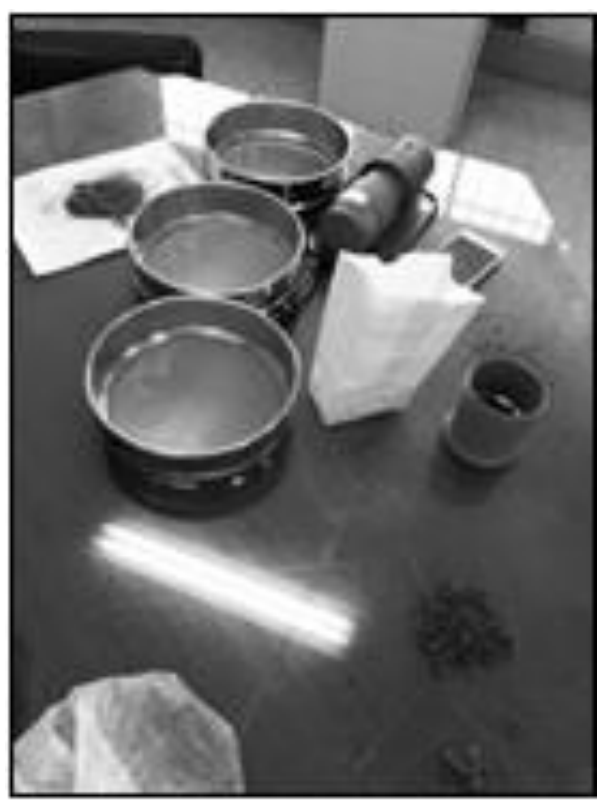

Fig. 11 Proceso triturado

Luego de conseguir la cantidad de madera necesaria para el ensayo, se procedió a tamizarlas, los resultados se muestran en la Tabla 6. 
Tabla 6. Valores tamizados de las maderas llevadas al laboratorio.

\begin{tabular}{cccc}
\hline \multicolumn{4}{c}{ GraDACIÓN MATERIAL } \\
\hline TAMIZ & UNIDAD & MELINA & PLÁ́sTICA \\
\cline { 3 - 4 } & & $\begin{array}{c}\text { PESO } \\
\text { RETEMIDO } \\
\text { (GR) }\end{array}$ & $\begin{array}{c}\text { PESO } \\
\text { RETENIDO } \\
\text { (GR) }\end{array}$ \\
\hline 25 & MM & 0 & 0 \\
\hline 19 & MM & 0 & 0 \\
\hline 12,5 & MM & 0,03 & 0 \\
\hline 300 & MICRA & 150,79 & 110,83 \\
\hline 150 & MICRA & 5,52 & 3 \\
\hline & FONDO & 2,84 & 0,12 \\
\hline & TOTAL & 159,18 & 113,95 \\
\hline
\end{tabular}

La tabla muestra que el peso total enviado al laboratorio de madera plástica fue de 113,95 gramos y el peso de madera natural enviado fue de 159,18 gramos, los cuales eran suficientes para empezar el conteo de $\mathrm{ufc} / \mathrm{g}$.

Los resultados y análisis fueron los siguientes:

Tabla 7. Resultados del análisis microbiológico

\begin{tabular}{cccc}
\hline ENSAYO & UNIDAD & MELINA & PLÁSTCA \\
\hline MOHOS Y LEVADURAS & UFC $/ \mathrm{G}$ & 90 & 40 \\
\hline
\end{tabular}

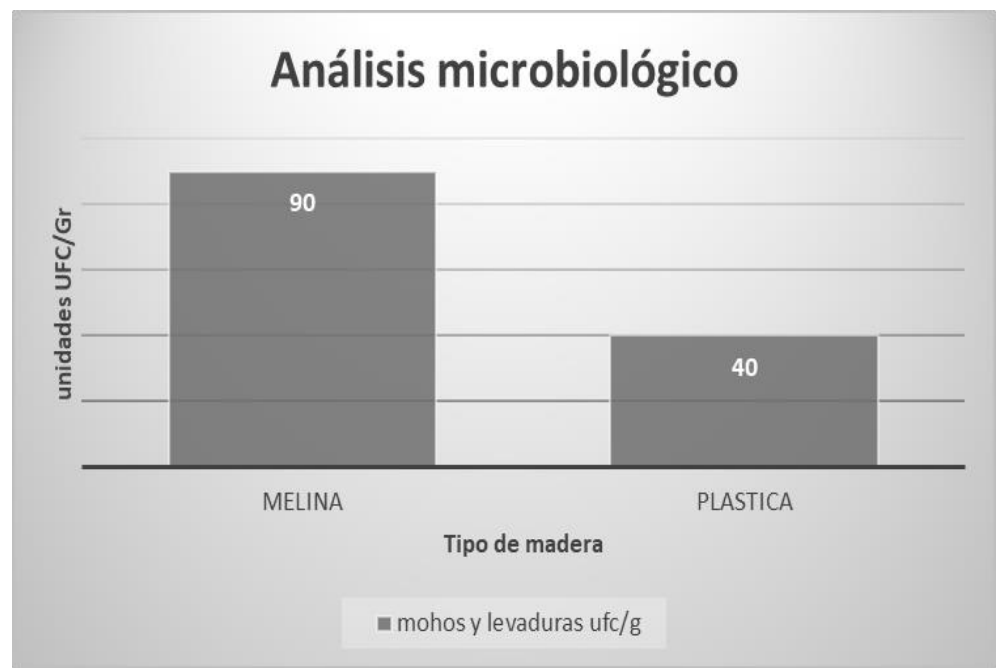

Fig. 12. Ufc/gr vs tipo de madera.

La madera natural demostró poseer la capacidad de retener 90 unidades formadoras de colonia en cada gramo de esta, y la madera plástica unas 40 unidades, es decir menos de la mitad de lo que es capaz de mantener la madera natural. 
Si bien, hay 40 ufc/g en la madera plástica, se afirma con total certeza que los microorganismos NO podrán reproducirse, debido a la falta de condiciones ambientales adecuadas, considerando que la madera plástica en condiciones normales, no supera un promedio de contenido de humedad del $0,5529 \%$, lo que se convierte en condiciones NO aptas.

Por otra parte, la madera natural puede mantener en 1 gramo 90 ufc, lo que por sí solo ya es alarmante, se añade que la humedad mínima para que un hongo pueda desarrollarse es de $13 \%$, luego de un $16 \%$ el hongo puede crecer de manera exponencial, y sabiendo que la humedad en condiciones normales de madera natural es de $24 \%$, se afirma que las 90 ufc encontradas se podrán desarrollar sin impedimento alguno, ya que la madera en cuestión ofrece las condiciones ambientales (humedad y sustrato) adecuadas para el desarrollo.

\subsubsection{Ensayo según NTC 1127 (insectos sociales)}

Se realizó el ensayo en los frascos de $50 \mathrm{ml}$ y frasco de $300 \mathrm{ml}$ [8] acondicionándoles el ambiente a condiciones de humedad, sustrato, temperatura y oxígeno similares a su medio, pero en ningún caso las termitas lograron adaptase al nuevo entorno y por ende, murieron en menos de 3 días. El ensayo se repitió más de 4 veces sin lograr la adaptación esperada. Se prosiguió con frascos de diversos tamaños buscando el mejor acondicionamiento para los organismos, pero todos los ensayos fracasaron [8].

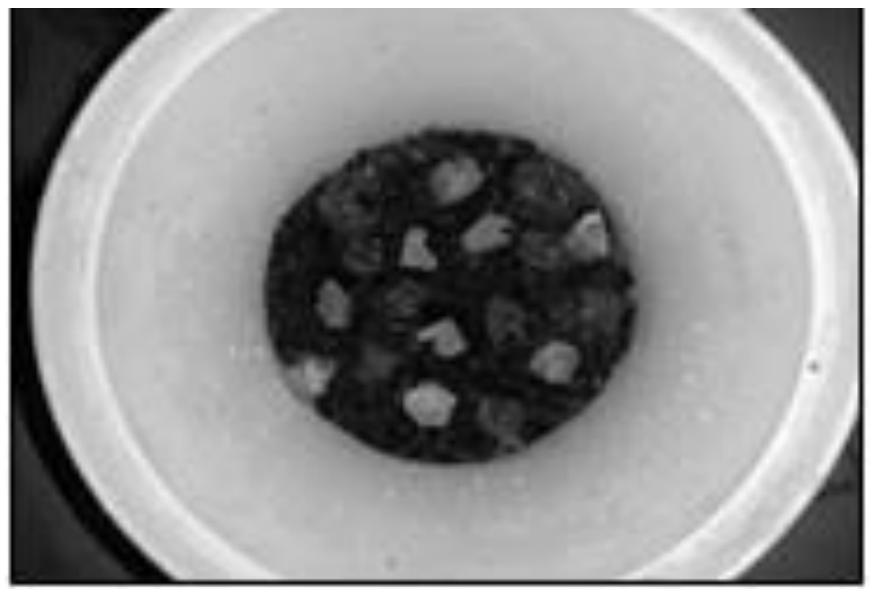

Fig. 13. Proceso de adaptación de las termitas.

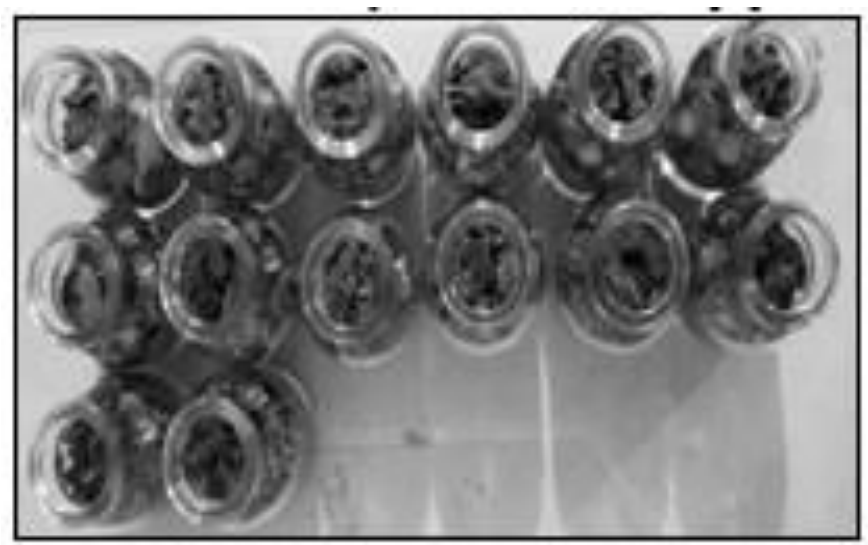

Fig. 14. Insectos sociales en frascos de $50 \mathrm{ml}$.

Se decidió crear una nueva metodología que combinara las normas NTC 1127 y la norma UNE EN 350-1 [9], la cual recibió el nombre de ensayo HÍBRIDO, analizado de la siguiente manera: 
Las condiciones favorables para termitas son estrictas y no se aconseja replicarlas en laboratorio. Los resultados por mes significaban trasladar y analizar un par de muestras, luego se desechaban, los resultados obtenidos por mes de cada madera fueron:

Tabla 8. Resultados de pérdida de peso en ensayo híbrido.

\begin{tabular}{ccc}
\hline \multicolumn{3}{c}{ \%PP (PÉRDIDA DE PESO) } \\
\hline & MELINA (GR) & PLASTICA (GR) \\
\hline 1MES & 24,0087 & 0,0039 \\
\hline 2 MES & 35,174 & 0,01985 \\
\hline 3 MES & 41,2403 & 0,0009 \\
\hline 4 MES & 49,4006 & 0,0009
\end{tabular}

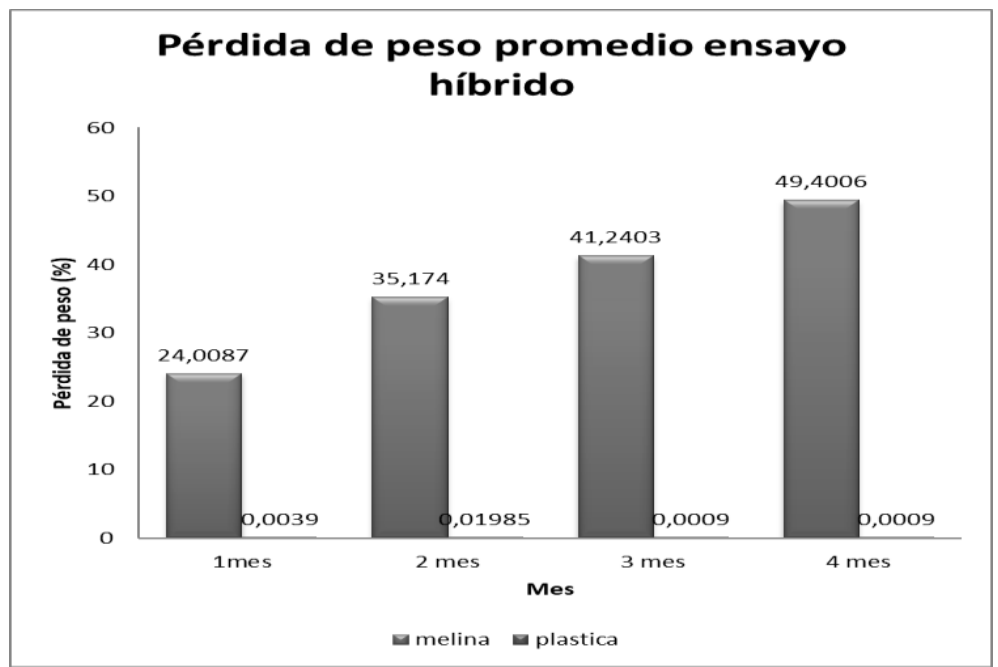

Fig. 15. Resultados del ensayo de durabilidad hibrido.

La diferencia de pérdida de peso entre la madera natural y la madera plástica es SIGNIFICATIVA.

La madera natural en 4.00 meses perdió casi la mitad de su peso y masa por ataque de termitas, y la madera plástica escasamente perdió un $0.01985 \%$ debido a factores ambientales encontrados en el medio, se descarta que haya sido por ataque de termitas debido a que la dieta de dichos insectos sociales es a base de celulosa, y la madera plástica al estar hecha de $100 \%$ polietileno la hace inmune a este tipo de ataques, muy al contrario de la madera natural que se compone de un $40 \%-50 \%$ de celulosa.

Lo anterior, se registra de acuerdo con lo contrastado en la Norma Técnica ASTM D-1238 [16]. Se corrobora la gran ventaja de la madera plástica frente a la natural en cuanto a todos los organismos xilófagos, ya que todos estos solo consumen la celulosa presente solo en la madera natural.

La madera natural se clasifica según la NTC 1127 como MODERADAMENTE RESISTENTE y según la UNE EN 350-1 como MEDIANAMENTE DURABLE. La madera plástica se clasifica según la NTC 1127 como ALTAMENTE RESISTENTE y según la UNE EN 350-1 como MUY DURABLE.

\subsection{Etapa 3: Evaluación de análisis de ciclo de vida ACV de las dos (2) maderas}

El análisis del inventario del ciclo de vida comparativo (ICV) para la estiba de madera natural elaborado en SIMAPRO 8.4.0 [10] fue el siguiente: 
Tabla 9. Inventario estiba natural ordinaria.

\begin{tabular}{ccc}
\hline ENTRADAS & CANTIDAD & UNDADES \\
\hline WÁTER, RIVER & 109500 & $\mathrm{~L}$ \\
\hline WOOD & 41,72 & $\mathrm{KG}$ \\
\hline SEED & 1 & $\mathrm{KG}$ \\
\hline TRANSFORMATION, FROM SEALED SOIL & 12.5 & $\mathrm{M} 2$ \\
\hline TRANSFORMATION, TO PERMANENT CROP, IRRIGATED, & 12.5 & $\mathrm{M} 2$ \\
EXTENSIVE & & $\mathrm{KG} / \mathrm{KM}$ \\
\hline TRANSPORT, COMBNATION TRUCK, AVERAGE FUEL MIX/US & 28.9 & $\mathrm{KG} / \mathrm{KM}$ \\
\hline TRANSPORT, COMRINATION TRUCK, GASOLINA POWERED/US & 41,72 & $\mathrm{KW} / \mathrm{H}$ \\
\hline ELECTRICTTY FROM HYDRO & 10 & \\
\hline
\end{tabular}

El inventario para la estiba de madera plástica elaborado en SIMAPRO 8.4.0 fue el siguiente:

Tabla 10. Inventario estiba plástica

\begin{tabular}{ccc}
\hline ENTRADAS & CANTIDAD & UNIDADES \\
\hline POLYETHYLENE HIGH DENSITY GRANULATE & 30 & $\mathrm{KG}$ \\
\hline LIQUID SILICONE & 0,7 & $\mathrm{KG}$ \\
\hline TRASINORT, COMBINATION TRUCK, GASOLINA POWERED & 22 & $\mathrm{KG} / \mathrm{KM}$ \\
\hline ELECTRICITY FROM HYDRO & 5 & $\mathrm{KW} /$ \\
\hline
\end{tabular}

El diagrama de flujos para una estiba de madera natural hecha en SIMAPRO 8.4.0 es el siguiente: 


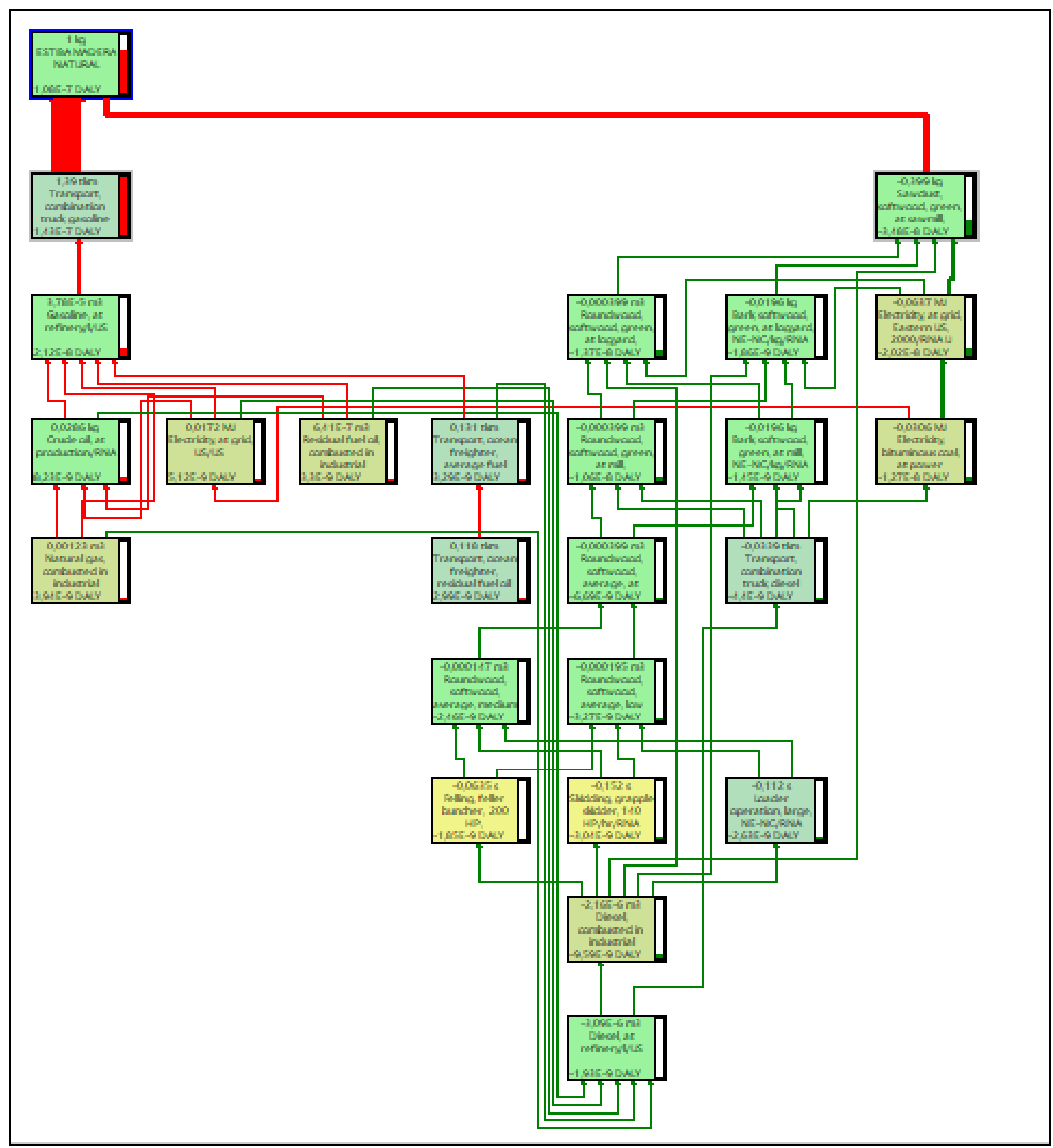

Fig. 16. Flujo de actividades para una estiba de madera natural

El análisis de impacto y caracterización, muestra que durante el ciclo de vida de una estiba de madera natural los impactos y sus porcentajes por entradas y salidas son ocupados casi en un $100 \%$ por la actividad de transporte impulsado por gasolina, lo que resultaría lógico, ya que los impactos ambientales generados por el factor saliente de esta actividad $\left(\mathrm{CO}_{2}\right)$ es negativo para cualquier ecosistema, el humo arrojado a las vías, el levantamiento de material particulado a las calles, etc. las categorías donde dicha actividad genera impactos son:

- Transporte impulsado por gasolina: TIG

- Cambio climático: 100\% TIG

- Toxicidad humana: $100 \%$ TIG

- Oxidante químico: $100 \%$ TIG

- Material particulado: $100 \%$

- Cambio climático en ecosistemas: 100\% TIG 
- Acidificación terrestre: $100 \%$ TIG

- Ecotoxicidad terrestre: $72 \%$ TIG

- Ecotoxicidad de agua dulce: $100 \%$ TIG

- Ecotoxicidad marina: $100 \%$ TIG

- Agotamiento de fósiles: $100 \%$ TIG

El diagrama de flujos para una estiba de madera plástica hecha en SIMAPRO 8 es:

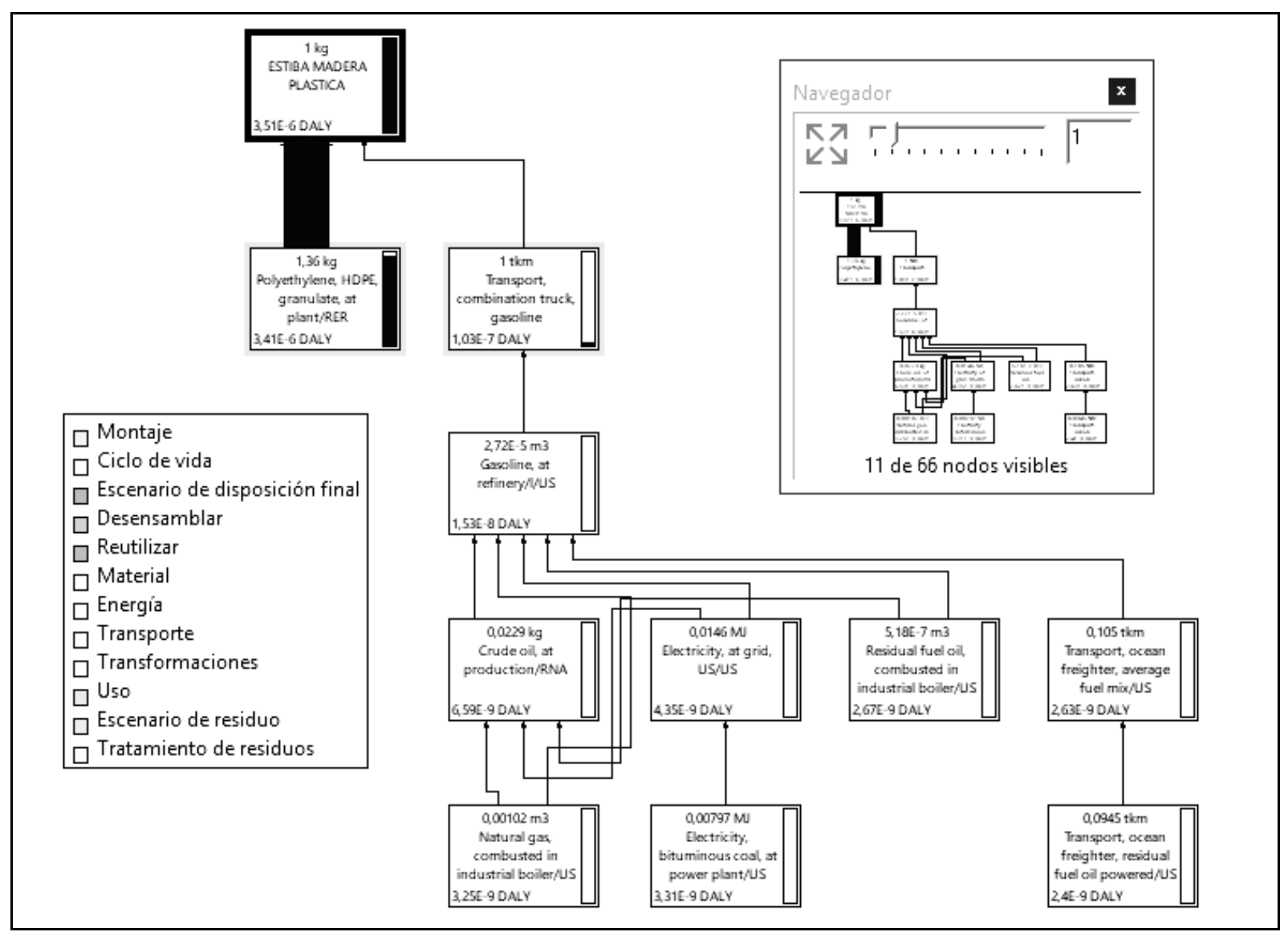

Fig. 17. Flujo de actividades para una estiba de madera plástica

El análisis de impacto y caracterización muestra que, durante el ciclo de vida de una estiba de madera plástica, los impactos generados por sus entradas y salidas se deben solo a 2 actividades, el manejo de polietileno de alta densidad y transporte impulsado por gasolina. El polietileno es un plástico muy usado en la actualidad, aunque en este proyecto se realiza para transformar los aspectos negativos del polímero en cuestión, en positivos para el ambiente y para la sociedad misma; los impactos ambientales negativos ocasionados por este polímero de etileno se deben al problema de la adecuada disposición final de los productos de polietileno de alta densidad y el hecho de que este polímero puede durar cientos de años antes de degradarse.

Por otra parte, y como ya se dijo anteriormente, el segundo factor, se debe a la liberación de $\mathrm{CO}_{2}$, material particulado y otros subproductos de esta actividad como el aceite para tracto mulas y demás, lo que ocasiona en menor medida impactos ambientales negativos.

- Manejo de polietileno de alta densidad: PAD

- Transporte impulsado por gasolina: TIG 
Las categorías donde dichas actividades generan impactos son:

- Cambio climático: 95\% PAD, 5\% TIG

- agotamiento de ozono: 99\% PAD, 1\% TIG

- Toxicidad humana: $66 \%$ PAD, $34 \%$ TIG

- Oxidante químico: 94\% PAD, 6\% TIG

- Material particulado: 94\% PAD, 6\% TIG

- Radiación ionizante: $100 \%$ PAD.

- Cambio climático en ecosistemas: 97\% PAD, 3\% TIG

- Acidificación terrestre: 94\% PAD, 6\% TIG

- Eutrofización de agua dulce: $100 \%$ PAD

- Ecotoxicidad terrestre: 99\% PAD, 1\% TIG

- Ecotoxicidad de agua dulce: 86\% PAD, 14\% TIG

- Ecotoxicidad marina: 84\% PAD, $16 \%$ TIG

- Ocupación de tierra agrícola: 100\% PAD

- Ocupación de tierra urbana: $100 \%$ PAD

- Transformación de tierra natural: 100\% PAD

- Agotamiento de metales: $100 \%$ PAD

- Agotamiento de fósiles: $99 \%$ PAD, $1 \%$ TIG

- Agotamiento de fósiles: $100 \%$

Como los factores de energías gastadas e impactos ambientales ya han sido evaluados en el ACV conceptual comparativo hecho en SIMAPRO 8.4.0, queda solo analizar la variable o factor TIEMPO, usando la herramienta OFFICETIMELINE en MICROSOFT POWERPOINT, simulando la elaboración de "cuna a tumba" de cada estiba de madera desde el año presente, los resultados y análisis son los siguientes: Estiba de madera natural: 100\% madera melina. Estiba madera plástica: 100\% polietileno de alta densidad.

De acuerdo con la Norma Técnica ISO-1133 [12] para la estiba de madera natural, el mayor tiempo durante su ciclo de vida es ocupado por la fase de extracción de materia prima, ya que, la madera melina debe crecer y desarrollarse durante mínimo 8 años para su primera poda, la segunda fase con más tiempo es la de uso y mantenimiento, la cual tarda 2 años, este cálculo es brindado por aserraderos oficiales quienes aseguran dicho tiempo para una estiba excelentemente cuidada. El mantenimiento en las estibas es casi nulo. Las otras fases no demoran más de 1 semana ya que tratan cosas simples y rápidas, como ensamblaje, transporte y disposición de desechos [15].

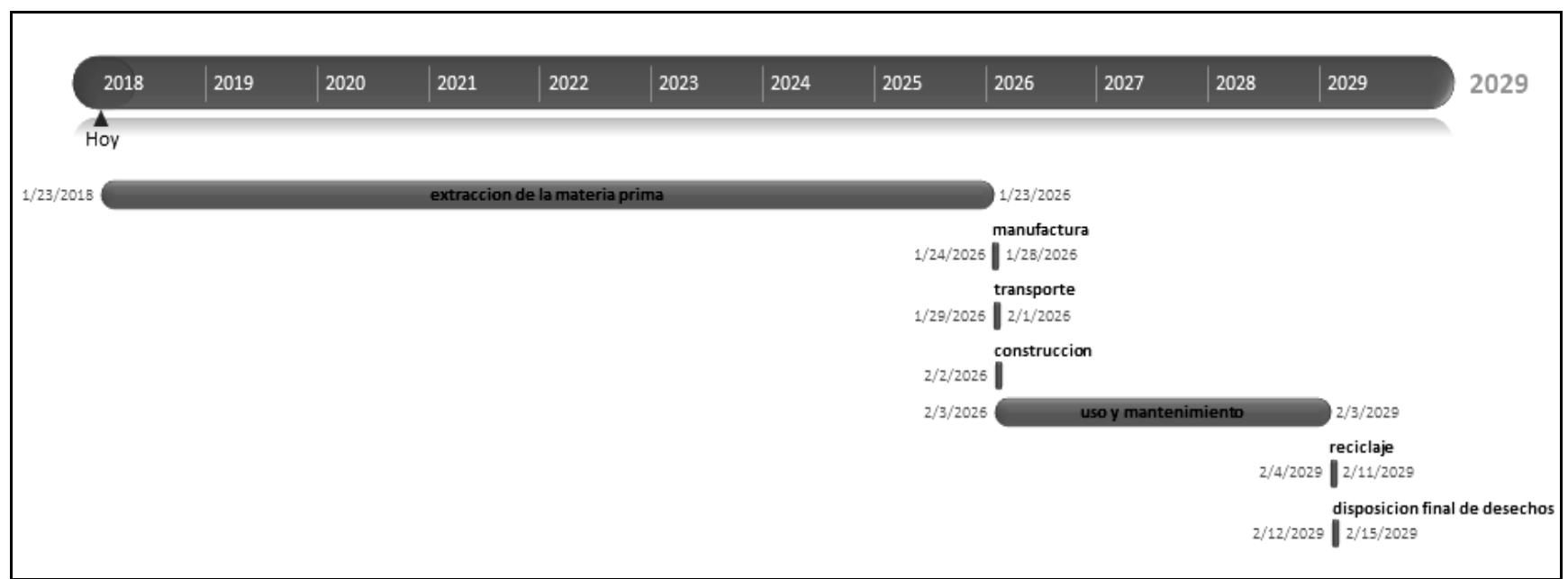

Fig. 18. Tiempo empleado en ciclo de vida de estiba de madera natural 
Para la estiba de madera plástica, es evidente que la fase de uso y mantenimiento es la que ocupa más espacio dentro de todo el ciclo de vida, esto se debe a la gran resistencia del polietileno de alta densidad en el ambiente, las otras fases del ciclo de vida no demoran más de unos pocos días ya que son actividades rápidas como transporte, ensamblaje, etc.

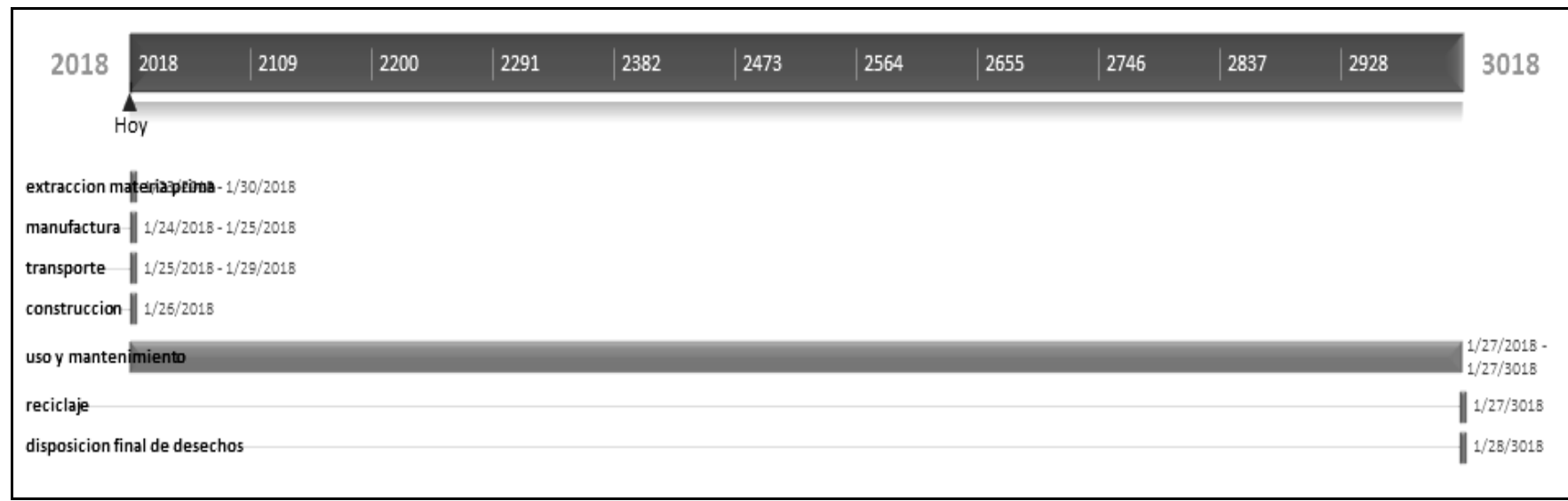

Fig. 19. Tiempo empleado en ciclo de vida de estiba de madera plástica

En cuanto a análisis comparativo, se hace notoria la diferencia de tiempos entre las 2 estibas, la estiba de madera natural cuya elaboración se simula a partir del año 2018, termina su ciclo de vida en el año 2029, por otro lado, la estiba de madera plástica comienza en el año 2018 y termina en el año 3018 . Son 989 años de diferencia, los cuales se explican debido a la naturaleza de la materia prima usada para su elaboración, amparado también en lo establecido en la Norma ASTM D 790-02 [13].

3.4. Etapa 4: Valoración económica (análisis de costos de producción) de los dos (2) tipos de estiba Los costos entre los dos tipos de maderas analizados arrojaron valores económicos diferentes, tal como se muestra en la fig. 20 la cual indica que el costo de producción de la unidad funcional en madera natural tiene un costo de $\$ 299.030,750$ pesos colombianos para el año actual y en madera plástica, un costo de $\$ 27.459 .000$ pesos colombianos.

Esta variación se debe principalmente al costo de extracción de materia prima en la madera natural, pues ocupa más del $60 \%$ en el costo total, y esto a su vez, se debe a la utilización de recursos ambientales y mano de obra a gran escala a diferencia de la plástica, la cual, en la misma fase en cuestión, puede ocupar un almacén de $5 \mathrm{~m} \times 5 \mathrm{~m}$. Se concluye que la madera plástica ocupa un porcentaje muy pequeño de dinero en comparación con la madera natural, por tanto, la idea de madera plástica se toma como la mejor opción para la inversión de dinero [11]. 


\section{Costo estiba madera natural Vs estiba madera plástica (188.5 estibas)}

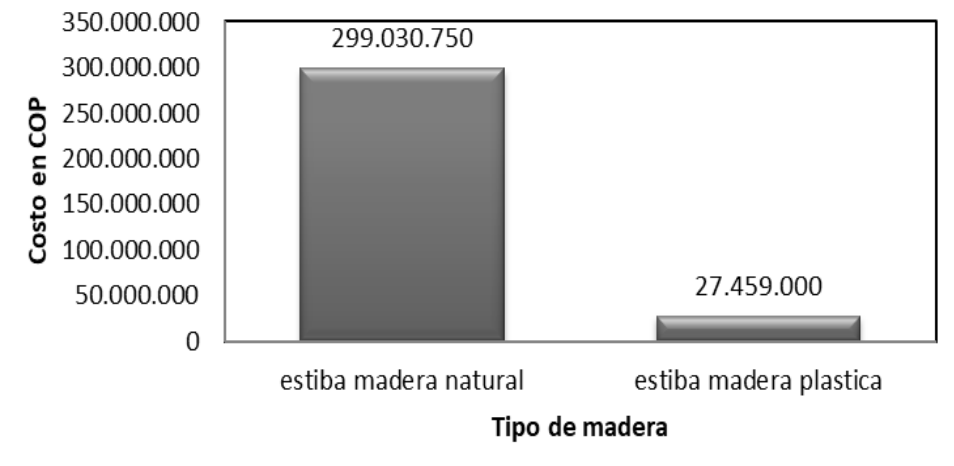

Fig. 20. Resultados de la valoración del ACV de ambos maderas.

\subsection{Etapa 5. Estudio de participación social}

Se aplicaron unas encuestas a una muestra de la población finita con permanencia total en el sector objeto de estudio y se concluyó que pese a la edad de los vendedores de la plaza MERCAUPAR la cual oscila entre 26 y 45 años y a pesar de tener un nivel de educación en promedio de básica secundaria (bachiller) se evidencia una mentalidad positiva y participativa en asuntos ambientales, lo que es conveniente para su futura participación en la construcción de estibas.

Los residuos sólidos inorgánicos más desechados por la población en cuestión es el plástico, en todas sus variaciones, lo que significa otro punto a favor del desarrollo óptimo del proyecto y viabilidad de este.

\section{DISCUSIÓN}

Con respecto a los ensayos físico - mecánicos, no hubo diferencia significativa en el de resistencia a la flexión entre los 2 tipos de madera, considerando que dicha diferencia entre ambas es de $1.8 \mathrm{MPa}$, con ventaja para la madera natural.

Los ensayos de humedad en condiciones normales y en tiempos de retorno de 3 y 10 años arrojaron valores de $24 \%, 39 \%, 80 \%$ y $1.6 \%, 1.1 \%, 0.8 \%$ en promedio para la madera natural y la madera plástica respectivamente, y debido a la incidencia de la humedad sobre las propiedades de la misma, se concluye que la madera plástica puede mantener sus propiedades físico mecánicas totalmente intactas en el tiempo de retorno máximo, al contrario de la madera natural quien por su naturaleza NO puede mantenerse en condiciones apropiadas en presencia de humedad.

El ensayo microbiológico demuestra la superioridad de la madera plástica sobre la madera natural, ya que la plástica puede contener $40 \mathrm{ufc} / \mathrm{gr}$, al contrario de la natural la cual posee más del doble (90 ufc/gr). En cuanto al ensayo híbrido, se concluye que el deterioro por insectos sociales en madera natural es superior que el deterioro de la madera plástica, ya que el ensayo arrojo valores de $49 \%$ y $0.0009 \%$ respectivamente.

\section{Con respecto al ACV}

La diferencia entre los impactos ambientales, hacia la salud humana y a los recursos son NOTORIOS y DISTANTES entre ambas maderas. SIMAPRO 8.4.0 logró calcular que las estibas de madera natural ocupan el $100 \%$ de dichas afectaciones, por otro lado, las estibas plásticas llegan apenas a un máximo de $47 \%$. Con lo que se afirma que ambientalmente la madera plástica es mejor que la madera natural. 


\section{Con respecto a la valoración económica}

El costo en COP de 188 estibas de madera plástica es de 27.459 .000 y para madera natural es 299.030.750, siendo la industria de madera natural mucho más costosa que su compañera. Se concluye entonces, que económicamente las estibas de madera plástica pueden implementarse mejor que las estibas de madera natural. Con respecto al estudio de participación social:Se encontró que la participación social para la ejecución del proyecto es positiva. La cantidad de RSI generados a diario son capaces de mantener la industria de la madera plástica.

Finalmente, el estudio de participación social hecho en MERCAUPAR LTDA. Para la futura construcción de estibas plásticas es socialmente viable.

Al trabajar con la versión DEMO de SIMAPRO 8.4.0 resultó imposible añadir algunas actividades en el ciclo de vida individual para ambas estibas, lo que hace que la información suministrada no sea $100 \%$ confiable a la hora de replicarse. Los argumentos justificados carecen de información obtenida de otros trabajos, ya que, como se aclaró al comenzar el artículo, NO existen pública y oficialmente trabajos de esta índole.

\section{CONCLUSIONES}

Los resultados obtenidos demuestran que los impactos ambientales de la construcción de estibas de madera natural son mucho mayores EN CRECES que los impactos relacionados con la construcción de estibas de madera plástica. Lo que nos conlleva a concluir que la alternativa de la madera plástica es mucho más amigable con el medio ambiente en general. En conclusión, es una excelente opción como alternativa para la construcción en los siguientes contextos:

Salud humana: El ACV comparativo, para la estiba plástica la afectación a la salud humana es del $2 \%$ y para la estiba natural del $100 \%$ lo que muestra la notoria diferencia entra impactos a la salud humana existentes entre las 2 estibas. Teniendo en cuenta los análisis de los ACV individuales por componente se dice que dichos resultados se deben al origen y consecuencias de los impactos a la salud humana encontrados, recordemos que para la estiba natural casi el $100 \%$ de los impactos estaban relacionados con el CO2 liberado que causa problemas severos y rápidos a la vida humana, inclusive llevándolos a la muerte bajo exposiciones frecuentes y duraderas, por otro lado, los impactos de la estiba plástica son muy poco frecuentes y necesitan tiempos de exposición casi permanentes para poder generar algún cambio a la salud humana.

Ecosistemas: El ACV comparativo, la estiba de madera plástica aporta un $2 \%$ en afectación a los ecosistemas, y la estiba de madera natural un $100 \%$ de impactos al medio ambiente. Esto se debe a que los impactos de la estiba plástica se relacionan con el polietileno de alta densidad en casi su totalidad, es decir, los plásticos mal dispuestos en zonas específicas que generan problemas ambientales. Por otro lado, está la afectación a los ecosistemas de la estiba natural, la cual está relacionada nuevamente con el CO2, siendo el $\mathrm{CO} 2$ uno de los más grandes contaminantes a nivel mundial y precursor del calentamiento global. Entonces, el nivel de afectación entre las 2 es de diferentes magnitudes, predominando la afectación de la estiba natural.

Recursos: EI ACV comparativo muestra que la afectación a los recursos por parte de la estiba de madera plástica es del $47 \%$, y la estiba natural del $100 \%$. Esto se debe a que en la estiba plástica solo intervienen materiales en casi un $100 \%$ reciclado (tomado de plantas de reciclaje) y otro pequeño porcentaje como aditivos y silicona dependiendo el método de construcción, que luego son fundidos y moldeados como perfiles. Por otra parte, la estiba de madera natural demanda en primera instancia, MADERA, madera que es extraída de cultivos de árboles maderables los cuales están listos para talar después de mínimo 7 años, si hablamos de árboles ordinarios, ya que para otros tipos de madera se necesitan mínimo 50 años, durante todo ese tiempo necesitan abundante agua para crecer y desarrollarse, luz solar, plaguicidas, suelo entre otros. Por ende, es evidente el gran consumo de recursos naturales por parte de las estibas de madera natural que las estibas de madera plástica. 


\section{AGRADECIMIENTOS}

Los autores expresan sus agradecimientos a los laboratorios de ensayos: V\&O Ingeniería SAS, Universidad Popular del Cesar y Bioindal Lab. Manifiestan reconocimiento especial a la empresa MERCAUPAR S.A por sus aportes para la realización de esta investigación.

\section{REFERENCIAS BIBLIOGRÁFICAS}

[1] M. V. Jara Salme. (2015). Diseño de una planta piloto para la obtención de granza de madera plástica a partir de polietileno reciclado con partículas lignocelulósicas,» Quito, Ecuador.

[2] L. Rincón Garzón, E. Rodríguez Carmona y A. Espitia Cubillos. (2016). Madera plástica. Un producto amigo del planeta. Semilleros, vol. 3, N. ${ }^{\circ}$ 5, pp. 4148.

[3] Ospina Restrepo, Carlos. (2014). Evaluación de las propiedades mecánicas de los perfiles extruidos a partir de mezclas de polímeros reciclados para la fabricación de estibas de maderas plásticas en MADERPOL S.A.S, 2014.

[4] Y. Martínez López, R. R. Fernández Concepción, D. A. Álvarez Lazo, M. García González y E. Martínez Rodríguez, «Evaluación de las propiedades físico-mecánicas de los tableros de madera plástica producidos en Cuba respecto a los tableros convencionales,» SciELO, 2014.

[5] Norma Técnica Colombiana/NTC- 663. Determinación de la resistencia a la flexión de maderas.

[6] Norma Técnica Colombiana NTC 206-1. Medición directa del contenido de humedad en la madera y materiales a base de madera.

[7] Norma Técnica Colombiana NTC 4132 Microbiología. Guía general para el recuento de mohos y levaduras. Técnica de recuento de colonias a $25^{\circ} \mathrm{C}$.

[8] Norma Técnica Colombiana NTC 1127 Maderas. Ensayo acelerado. Durabilidad natural

[9] UNE EN 350-1 UNE-EN 350-1:1995 Guía para los principios de ensayo y clasificación de la durabilidad natural de la madera.

[10] SIMAPRO 8.4.0. Análisis del Ciclo de Vida y uso del Software SIMAPRO.

[11] Ibarra y Asociados, valoración económica del impacto ambiental del manejo de residuos sólidos orgánicos, 2016.

[12] Norma Técnica ISO-1133 “Determinación del Índice de Fluidez en Termoplásticos”.

[13] Norma ASTM D 790-02, Standard Test Methods for Flexural Properties of Unreinforced and Reinforced Plastics and Electrical Insulating Materials, Vol. 8.01, Philadelphia (EE.UU.): American Society for Testing and Materials.

[14] NTC 3545 Norma Técnica Colombiana. Maderas y Adhesivos de resinas sintéticas fenólicas y amino plásticas para madera contrachapada, 1993.

[15] Mejía Mesa, Gonzalo Alberto. Gómez López, John Santiago. "Los Desechos Generados por la Industria Bananera Colombiana". En: Seminario Internacional; Gestión Integral de Residuos Sólidos y Peligrosos, Siglo XXI, 2017.

[16] Norma Técnica ASTM D-1238. Determinación de fluidez en masa (MFR) y del índice de fluidez en volumen (MVR).

[17] O. F. Delgado y J. A. Medina, «Extrusión de perfiles espumados de madera plástica,» Revista de Ingeniería Universidad de los Andes, vol. 2003, nº 18, pp. 5663, 2013. 